\title{
Therapeutic Strategies for Liver Abscess
}

\author{
Jie Zhang ${ }^{1,2}$, Chao Jun Kong ${ }^{1}$, Zhong Jia ${ }^{2^{*}}$, Liang Liang Fang ${ }^{1}$ \\ ${ }^{1}$ Hangzhou First People's Hospital, Nanjing Medical University Affiliated Hangzhou Hospital, Hangzhou 310006, \\ China \\ $2^{2 *}$ Department of Hepatopancreatobiliary Surgery, Hangzhou First People's Hospital, Hangzhou Huansha Road 261, \\ City of Hangzhou 310006, Zhejiang Province, China
}

Accepted on March 20, 2018

\section{Clinical Image}

This is the case of a 36-year-old man suffered from high body temperature and subcostal pain, with leukocytosis, elevated C-reactin protein and pro-calcitonin. Further enhancedcomputed tomography of abdomen (Figure 1) suggested the diagnosis of liver abscess. The optimal treatment option had better abide by step-up approach as follows: Besides effective broad-spectrum antibiotics, minimally invasive procedures, including earlier ultrasound-guided percutaneous catheter puncture/drainage and/or nasobiliary drainage via ERCP route have been worldwide applied first. But when and if all of these are inadequate or chronic liver abscesses with thick wall or intrahepatic stone disease co-existing or liver atrophy emerging and other special situations, surgical liver resection still remains its value. In practice, its potential etiology should be removal. Of note, if liver abscess is not so "ripe" to promise effective drainage, decompression of a "raw "actually also contributes to activate general condition. Liver abscess was completely disappearing after patient received percutaneous catheter drainage combined with antibiotic and appendectomy later. On review, three month after discharge, the patient recovered with no evidence of recurrence.

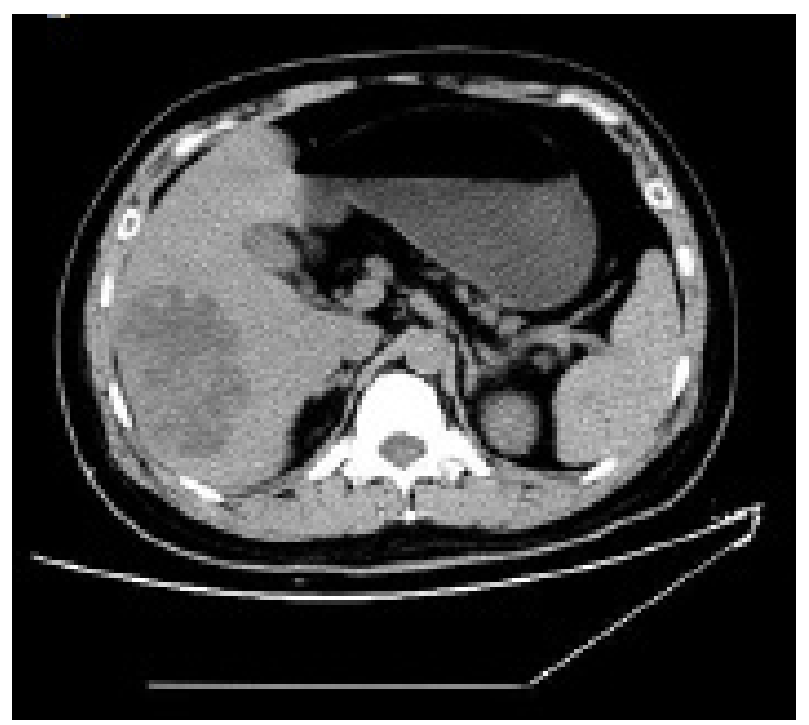

Figure 1. Enhanced-computed tomography of abdomen

\section{*Correspondence to:}

Zhong Jia

Department of Hepatopancreatobiliary Surgery

Hangzhou First People's Hospital

Hangzhou Huansha Road 261

City of Hangzhou 310006

Zhejiang Province

China

Tel: + 86-13958114181;

Fax: + 86-0571-87914773

E-mail: jiazhong20058@hotmail.com 